\title{
Local Oscillator Phase Noise Influence on Single Carrier and OFDM Modulations
}

\author{
Vitor Fialho $^{1,2}$, Fernando Fortes ${ }^{2,3}$, and Manuela Vieira ${ }^{1,2}$ \\ ${ }^{1}$ Universidade Nova de Lisboa - Faculdade de Ciências e Tecnologia- DEE \\ Monte da Caparica 2829-516, Lisbon, Portugal \\ ${ }^{2}$ Instituto Superior de Engenharia de Lisboa - ISEL \\ Rua Conselheiro Emídio Navarro,1 1959-007-Lisbon, Portugal \\ ${ }^{3}$ Instituto das Telecomunicações \\ Av. Rovisco Pais, 11049 - 001 Lisbon - Portugal \\ \{vfialho, ffortes\}@deetc.isel.ipl.pt, mv@isel.pt
}

\begin{abstract}
This paper describes the influence of local oscillator phase noise on single and multi-carrier modulations schemes. The oscillator has the main role on modulation and demodulation process of the radio frequency channels. The synchronization of the entire system depends on the accuracy of this circuit. This work is based on the simulation of these two scenarios under influence of a non-ideal oscillator. The output of the simulation scenario results of error vector magnitude, bit error rate and symbol error rate. The relation of these metrics with the local oscillator phase noise allows the performance estimation the global system. A simple phase noise model is presented with configurable power spectral density and offset frequency.
\end{abstract}

Keywords: phase noise, radio frequency transceiver, error vector magnitude.

\section{Introduction}

The local oscillator (LO) is one of the building blocks of every radio frequency (RF) front-end. This device is responsible, with the mixer, for the up and down conversion of a specific channel. However the modulation and demodulation processes suffer additional effects caused by the electronic circuits and the transmission channel itself. Common standards such as IEEE 802.11, [1] IEEE 802.16[2] do not present electrical specifications of the RF building blocks but system level specifications. The RF transmitter in several standards is specified by the channel centre frequency, bandwidth, spectral mask and error vector magnitude (EVM). The RF receiver is specified with bit error rate (BER) dependency on the received RF channel power (sensitivity), which depends on the received signal to noise ratio (SNR) and noise degradation due to noise figure and LO phase noise [3].

The digital modulation scheme used on these several standards depends on the relation of BER and SNR. Therefore, according to the channel noise conditions, the chosen modulation may change from quadrature phase shift keying (QPSK) to quadrature amplitude modulation (QAM). 
This scatter representation of the in-phase (I) and quadrature (Q) channels enables the evaluation of the global system performance, since RF impairments have a typical behavior on the received symbols [4]. Since the wireless transmission is a frequency selective fading channel, the most common standards adopted orthogonal frequency division multiplexing (OFDM) instead of a single carrier transmission. The main difference of both techniques is that OFDM use of a multicarrier technique which divides the entire bandwidth of the channel into smaller sub bands, around each subcarrier. This allows the study of LO influence on both systems and estimate the impact on the typical base band figures of merit.

With the upcoming of the 3GPP Long Term Evolution (LTE) single carrier and OFDM techniques are used on the same equipment. For the downlink transmission it is used OFDM in order to minimize multipath fading and inter-symbolic interference (ISI). In the uplink it is used a single carrier modulation technique since PAPR values are lower compared with OFDM.

Since the main study of this work is the influence of LO phase noise on the radio channel, only a generic OFDM signal is discussed. Cyclic prefix, interleaving and windowing [5] are not discussed in this work.

\section{Internet of Things}

The identification of several equipments, also know as things, via Internet, need to have a build in RF device. This device allows communication between the equipment and a given gateway for further identification. Therefore the study of LO performance on the global RF front-end has an important role on this area.

\section{Global System Description}

As depicted in figure 1 the RF front end, (transmitter and receiver), the signal delivered to, and from the mixer, are mapped in IQ channels. The channels content itself, and the modulation method used, single carrier or OFDM, does not have a direct influence on RF front end behavior. However, the RF impairments from the building blocks will influence the global system performance, namely the LO phase noise [4]. The base band signal is formatted by the base band processor that converts a digital to an analog signal for the up conversion.

\subsection{Base Band Signal}

In this sub-section it is presented the generic block diagram of a single carrier and OFDM signal generation.

Figure 2 presents the transmitter and receiver of a single carrier system based on IQ channels, where the digitized binary data stream is coded in QPSK or M-QAM. This is a straightforward way of mapping the symbols on an IQ channel. The square root raised cosine is used on the transmitter and receiver and allows, simultaneously, the control of the signal bandwidth and eliminate the inter-symbolic interference (ISI) [2][3][4]. 


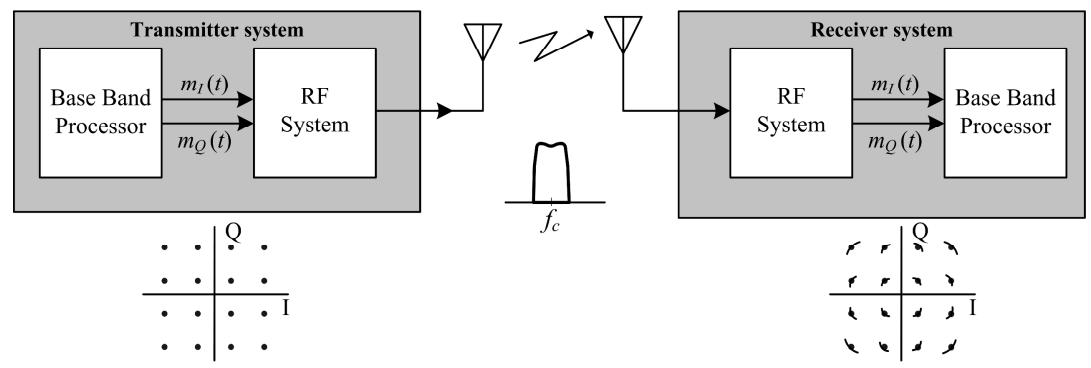

Fig. 1. Communication system based on IQ signals under phase noise influence

After the filtering process, the resulting base band analog signals, composed by $m_{I}(t)$ and $m_{Q}(t)$ are up converted and centered on the desired frequency channel, defined by the local oscillator of the transmitter $\left(\mathrm{LO}_{\mathrm{TX}}\right)$, included on the RF transmitter front end block. The maximum occupied bandwidth corresponds to the symbol frequency. After the transmitter and receiver synchronization process is complete, the data channel content is down converted for filtering and decoding.

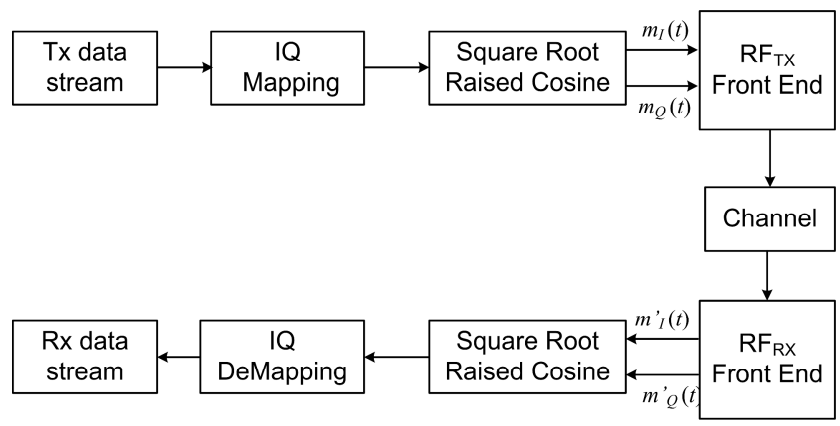

Fig. 2. Single carrier base band IQ signal generation

Figure 3 presents the transmitter and receiver OFDM block diagram. This is based on a multi-carrier modulation technique which uses orthogonal sub-carriers to transmit the base band data. In the frequency domain, since the bandwidth of a subcarrier is designed to be smaller than the coherence bandwidth of the channel, each sub-channel is seen as a flat fading channel which simplifies the equalization process [5]. In the time domain, by splitting a high-rate data stream into a number of lowerrate data streams that are transmitted in parallel, OFDM resolves the problem of ISI in wide band communications.

The output of the digital modulation is converted from serial to parallel streaming, composed by $\mathrm{X}_{0}$ to where $\mathrm{X}_{n}$ where $n$ represents the IFFT dimension. The detailed explanation of the global system and building block optimizations is described in several works [5][6][7]. Since OFDM is based on orthogonal sub-carriers, where the sub-channel information is centered on, it is important to have a correct knowledge of the LO phase noise influence on the entire bandwidth, and subsequent relation with base band metrics, such as EVM, BER and symbol error rate (SER) [4]. 


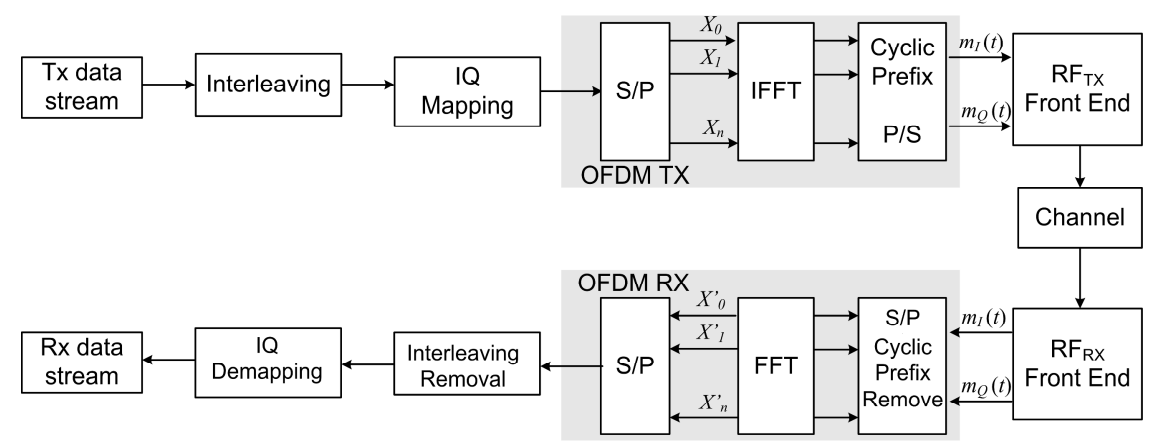

Fig. 3. Generic OFDM block diagram topology

\subsection{Radio Frequency Front End}

Figure 4 presents a typical RF front end based on IQ channels. The transmitter is composed by the mixer and $\mathrm{LO}_{T X}$ generating the $s_{R F}(t)$ centered on the desired transmitting frequency.

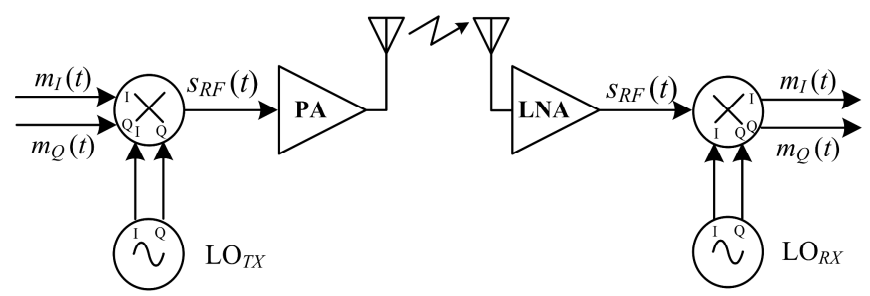

Fig. 4. Radio frequency front end based on IQ channels

The IQ base band signals are up-converted by the mixer and $\mathrm{LO}_{T X}$ to the desired RF channel. The power amplifier (PA) amplifies the signal to the desired transmission power. The receiver is composed by the low noise amplifier (LNA) which amplifies the RF band in which the desired RF channel is located. The filtered channel is downconverted by the mixer and $\mathrm{LO}_{R X}$ to an IQ base band signal for prior processing.

The RX blocks presented in figure 2 and figure 3 can be described as the RF front end presented in figure 4. As depicted this block is independent of how the base band signals are composed, as discussed in 3.1.

The RF impairments will be reflected on the received (demodulated) channel. The PA non-linearities and consequent peak peak-to-average power ratio (PAPR) is discussed by several authors [5][6][7]. As depicted, the common RF building block presented on the transmitter and receiver is the LO. Therefore it is important to study the influence of phase noise on the global system performance. Since the base band information is mapped on IQ channel it is possible to infer the global system behavior based on EVM and consequently on SER and BER.

Leeson [8], and the subsequent authors [9] established well known models that describe the phase noise variation at a specific frequency carrier offset. RF designers, 
specifically the LO designers, focus their optimization on specific phase noise values, typically at $1 \mathrm{MHz}$ and $10 \mathrm{MHz}$, depending on the system application[10]. This is useful for the oscillator characterization itself. However, when the oscillator up or down converts a channel, this characterization is not complete, since the phase noise variation will not be constant in all the channel bandwidth. Therefore it is important the have the knowledge of phase noise integrated power on the channel bandwidth and not at a specific offset frequency.

\section{Simulation Scenario}

In this section the simulation scenarios for both systems are presented, as well as the oscillator phase noise model developed for this work.

The single carrier and OFDM systems are implemented in MatLab-Simulink with a channel bandwidth of $1 \mathrm{MHz}$.

The simulation scenario for the single carrier modulation is based on the figure 2 topology. The IQ mapping is 16-QAM with a symbol frequency of $1 \mathrm{MHz}$. The square-root raised cosine has a roll-off factor of 0.2 .

OFDM simulation scenario is based on the topology presented in figure 3 with a FFT and IFFT dimension of 64 points. Therefore the final symbol rate is given by $f_{\text {samp }} / 64$ with a configurable number of sub-channels [1].

The RF front end is based on an oscillator with configurable phase noise power, as presented in section 4.1 .

\subsection{Phase Noise Model}

A generic oscillator with phase noise is expressed by (1), where $\theta_{\text {err }}(t)$ corresponds to a Gaussian distributed values with variance $v_{\text {noise }}{ }^{2}$. For low $\theta_{\text {err }}(t)$ values, (1) can be expressed by (2).

$$
\begin{gathered}
\mathrm{c}(\mathrm{t})=\cos \left(2 \pi f_{c} t+\theta_{\text {err }}(t)\right)+j \sin \left(2 \pi f_{c} t+\theta_{\text {err }}(t)\right) . \\
\mathrm{c}(\mathrm{t})=\cos \left(2 \pi f_{c} t\right)+j \sin \left(2 \pi f_{c} t+\theta_{\text {err }}(t)\right) .
\end{gathered}
$$

The developed phase noise model is presented in figure 5. The output values of the Gaussian noise generator are parameterized by $v_{\text {noise }}{ }^{2}$ and applied to a low pass filter with configurable cut-off frequency, $f_{\text {cut }}$. This allows the evaluation of the integrated phase noise power with limited bandwidth [8]. This configuration allows further studies of filtering technique in order to perform noise shaping.

The first order low pass filter is defined is $s$ domain as (3), where $a$ corresponds to the pole frequency. Since the implemented simulation scenario is based on a discrete environment, it is necessary to obtain an equivalent transfer function that represents the same filter but in a discrete domain. The approximate equivalence from Laplace domain and $\mathrm{z}$ domain is given by (4), where $T_{s}$ represents the simulation sampling time. 


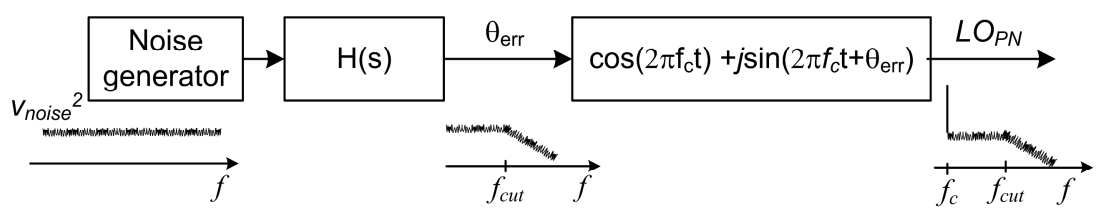

Fig. 5. Local oscillator phase noise model with configurable power and cutoff frequency

$$
\begin{gathered}
H(s)=\frac{1}{s+\mathrm{a}} \\
H(z)=H(s) \mid \begin{array}{c}
s=\frac{2}{T s} \cdot \frac{1-z^{-1}}{1+z^{-1}}=\frac{T s}{2+a T s} \cdot\left[\frac{z+1}{z+\frac{a T s-2}{a T s+2}}\right]
\end{array}
\end{gathered}
$$

\section{$5 \quad$ Simulation Results}

In this section the simulations results of single carrier and OFDM are presented under LO phase noise influence.

In order to quantify the error on both modulations techniques EVM is used since it can, without decoding the channel content, establish a relation between the transmitted and received signals, $s_{T X}[\mathrm{n}]$ and $s_{R X}[\mathrm{n}]$, respectively, as described by (5).

$$
E V M=\sqrt{\frac{1 / N \sum_{n=1}^{N}\left(s_{R X}[n]-s_{T X}[n]\right)^{2}}{1 / N \sum_{n=1}^{N}\left(s_{T X}[n]\right)^{2}}}
$$

In order to simplify the EVM extraction, both signals are normalized to their squareroot mean power. Therefore, the maximum EVM obtained is coincident with the $v_{\text {noise }}$ with a power spectral density (PSD) is given by $v_{\text {noise }}^{2} / f_{\text {samp }}$.

Since the EVM express the error, this can be estimated based on noise PSD. However, this depends on the number sub-carriers (NC), as expressed by(6).

$$
E V M=\sqrt{v_{\text {noise }}^{2} / f_{\text {samp }} \cdot N C \cdot \Delta_{f}}
$$

Figure 6 (a) presents the EVM variation for the OFDM system with $f_{\text {cut }}$ frequency greater than $f_{\text {samp }} / 2$ (equivalent to a constant phase noise) for different $v_{\text {noise }}$ values corresponding to the EVM values obtained by (6). Since the variation of EVM for the presented values are very small and similar, it is presented the $\log _{10}(\mathrm{EVM})$ instead of EVM itself. 


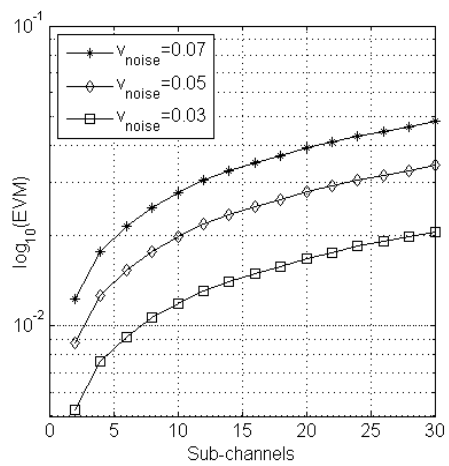

(a)

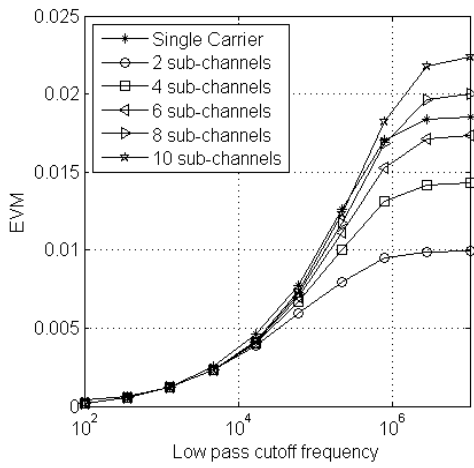

(b)

Fig. 6. (a) Variation of $\log _{10}(\mathrm{EVM})$ variation with OFDM sub-channels for different $v_{\text {noise }}$ values; (b) Variation of EVM in function first order low pass filter cutoff frequency for multiple sub-channels

Figure 6 (b) presents the EVM variation for both modulations techniques with $v_{\text {noise }}=50 \mathrm{mV}$. For each sub-channel EVM is obtained in function of $f_{\text {cut }}$ sweep of the low pass filter. As depicted for $f_{\text {cut }}$ values bellow $100 \mathrm{kHz}$, the EVM values for both modulation schemes are identical.

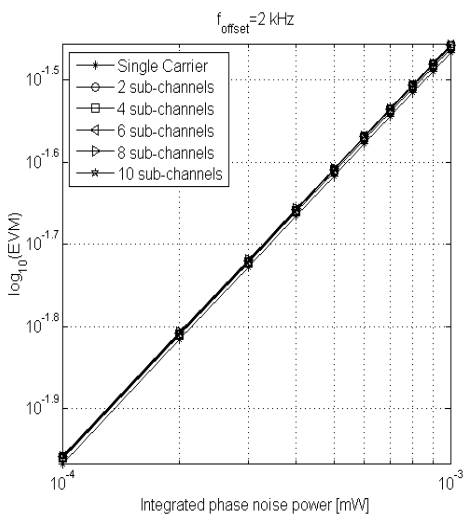

(a)

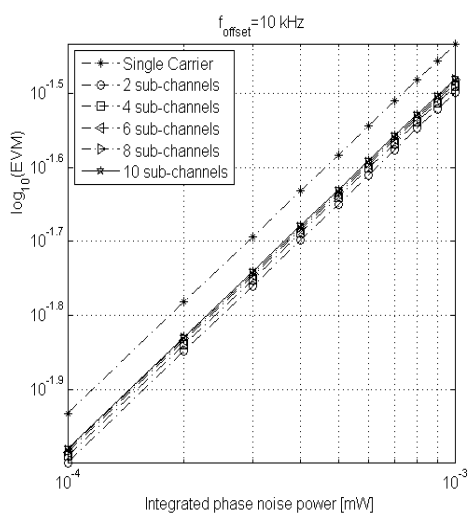

(b)

Fig. 7. EVM evolution for different carrier offset frequency: (a) $f_{\text {offset }}=2 \mathrm{kHz}$; (b) $f_{\text {offset }}=10 \mathrm{kHz}$

Figure 7 (a) and (b) presents EVM variation in function of integrated phase noise power for two different carrier offset frequency. For lower carrier offset frequency, the EVM difference between single carrier and OFDM is identical.

For offset frequency above $10 \mathrm{kHz}$, as presented in figure 7 (b) the number OFDM sub-channels has influence on EVM, and the single carrier system has a greater degradation then OFDM 


\section{Conclusions}

In this work a comparison between single carrier and OFDM modulation under the influence of local oscillator phase noise is presented.

A local oscillator phase noise model was presented. This model allows the configuration of the integrated phase noise power for a specific bandwidth. The final system performance is obtained by EVM. By the simulation results, it is possible to verify that the increase of sub-carriers on OFDM, for a specific phase noise may cause a greater degradation on EVM, and consequently on BER. The presented simulations allow concluding that, for oscillators with high phase noise, single carrier and OFDM has the same behavior. Therefore it is relevant, in the project of a RF system, the knowledge of the integrated phase noise power for several carrier offset frequency in order to optimize the global system performance.

This technique allows the study of different filter topologies with the purpose of perform noise shaping.

\section{References}

1. IEEE Standard for Wireless LAN Medium Access Control (MAC) and Physical Layer (PHY) Specifications: High Speed Physical Layer Extension in the 2.4GHz, IEEE Standard 802.11b-1999

2. IEEE 802.16. IEEE Recommended Practice for Local and metropolitan area networks Coexistence of Fixed Broadband Wireless Access Systems

3. Fortes, F., et al.: RF Receiver Front End for $28.5 \mathrm{GHz}$ applications on a $70 \mathrm{GHz}$ FT SiGe BiCMOS process. Wiley Microwave and Optical Technology Letters 52(3), 736-740 (2010)

4. Fialho, V., et al.: Test Setup for Error Vector Magnitude Measurement on WLAN Transceivers. In: 19th IEEE International Conference on Electronics, Circuits, and Systems-ICECS (December 2012)

5. Wang, et al.: OFDM or Single-Carrier Block Transmissions? IEEE Transactions on Communications 52(3) (March 2004)

6. Armstrong, J., et al.: OFDM for Optical Communications. Journal of Lightwave Technology 27(3) (Febraury 2009)

7. Jamal, M.: Study of Multiple Access Schemes in 3GPP LTE OFDMA vs. SC-FDMA. In: IEEE International Conference on Applied Electronics, AE (2011)

8. Leeson, D.B.: A Simple Model of Feedback Oscillator Noise Spectrum. IEEE Proceedings on IEEE 54(2), 329-330 (1966)

9. Lee, T.H., Hajimiri, A.: Oscillator Phase Noise: A Tutorial. IEEE Journal of Solid-State Circuits 35(3), 326-336 (2000)

10. Muh-Dey, W., Sheng-Fuh, C., Xu-Wei, L., Gyn-Wei, K.: Phase Noise Optimization of CMOS VCO with Double-Harmonic-Tuned LC Tank. In: IEEE European Microwave Conference, pp. 1054-1057 (October 2010) 